The submitted manuscript has been created by the University of Chicago as Operator of Argonne National Laboratory ("Argonne") under Contract No. W-31-109-ENG-38 with the U.S. Department of Energy. The U.S. Government retains for itself, and others acting on its behalf, a paid-up, nonexclusive, irrevocable worldwide license in said article to reproduce, prepare derivative works, distribute copies to the public, and perform publicly and display publicly, by or on behalf of the Government.

\title{
The Structure of High-Z Nuclei from Studies with Gammasphere at ATLAS
}

\author{
R.V.F. Janssens ${ }^{a}$

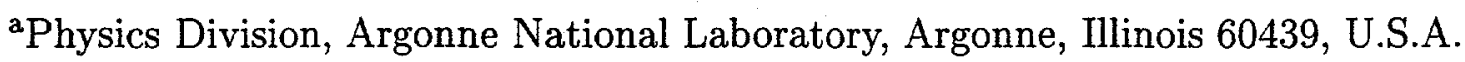

This presentation reviews one of the main research programs carried out with the Gammasphere array at ATLAS. Taking advantage of the availability of very heavy beams on the one hand, and of the coupling of Gammasphere with the Fragment Mass Analyzer on the other, it was possible to gain new insights into the properties of actinide and transfermium nuclei. The impact of octupole correlations on the structure of the Plutonium isotopes and the role of shape driving shell effects on the structure and on the formation of Nobelium nuclei is discussed.

\section{INTRODUCTION}

The Gammasphere array [1] was moved from the 88-Inch cyclotron at Lawrence Berkeley National Laboratory, where it was originally assembled, to the ATLAS superconducting linear Accelerator at Argonne National Laboratory with the aim of directing the power of the instrument towards exotic nuclei far from stability. This goal was made possible in large part by the coupling of the device with the Fragment Mass Analyzer (FMA) [2], an instrument tailored towards the detection and identification of evaporation residues in the presence of large backgrounds due to other processes. In addition, the wide range of beams available from the ATLAS accelerator and the exceptional timing characteristics made many novel uses of Gammasphere possible.

One direction of research focused on the properties of the heaviest nuclei, e.g. the actinides and the transfermium nuclei. It was possible to investigate the properties of two isotopes of Nobelium, element 102. Like the super-heavy elements, Nobelium owes its stability to the presence of strong shell effects. In ${ }^{253,254}$ No discrete line spectroscopy as well as calorimetry measurements were performed for the first time. The results show these heavy, fissile nuclei to be more stable against rotation than had previously been thought. In addition, from the entry distributions, information was obtained on the reaction mechanism(s). The results may well have implications for the synthesis of superheavy elements.

The actinide nuclei have often been regarded as some of the best candidates for studies of collective phenomena. Not only are these nuclei deformed, but their low-energy spectra also exhibit properties associated with vibrational degrees of freedom. Hence, the opportunity exists to study the interplay between vibrations and rotations. A set of recent Coulomb excitation measurements in $\mathrm{Pu}$ isotopes and in neighboring nuclei has focused on the unexpected impact of octupole correlations. Striking differences were found 


\section{DISCLAIMER}

This report was prepared as an account of work sponsored by an agency of the United States Government. Neither the United States Government nor any agency thereof, nor any of their employees, make any warranty, express or implied, or assumes any legal liability or responsibility for the accuracy, completeness, or usefulness of any information, apparatus, product, or process disclosed, or represents that its use would not infringe privately owned rights. Reference herein to any specific commercial product, process, or service by trade name, trademark, manufacturer, or otherwise does not necessarily constitute or imply its endorsement, recommendation, or favoring by the United States Government or any agency thereof. The views and opinions of authors expressed herein do not necessarily state or reflect those of the United States Government or any agency thereof. 


\section{DISCLAIMER}

Portions of this document may be illegible in electronic image products. Images are produced from the best available original document. 
between the yrast and negative parity bands in ${ }^{238-240} \mathrm{Pu}$ and those in ${ }^{241-244} \mathrm{Pu}$, which can be linked to variations with mass of the strength of octupole correlations. At the highest spins, ${ }^{238-240} \mathrm{Pu}$ are found to exhibit properties associated with stable octupole deformation, suggesting that a transition with spin from a vibration to stable deformation may have occurred.

\section{SHELL STABILIZED HIGH-Z NUCLEI}

\subsection{Yrast Spectroscopy and Deformation of ${ }^{253,254}$ No}

The heaviest nuclei, with $Z>100$, are at the limit of Coulomb instability, and would be unstable against spontaneous fission but for a large shell-correction energy, which leads to additional binding and creates a sizable fission barrier. The relative stability of these very heavy elements is a striking manifestation of shell structure in nuclei. Its importance was highlighted again recently with reports of the detection of elements 114, 116 and $118[3,4]$. Theoretical studies have indicated that these shell effects lead to an island of spherical superheavy elements [5] based on doubly closed neutron $(N=184)$ and proton $(Z=114)$ shells. In contrast, the observed stability of lighter transfermium nuclei with $Z$ up to 112 is explained [6] by invoking the ability of these nuclei to deform; e.g. the shell-correction energy is the largest for a sizable quadrupole deformation with contributions from higher order multipole moments.

Two isotopes of element $102,{ }^{254}$ No and ${ }^{253}$ No have been studied with Gammasphere and the FMA using the Recoil Decay Tagging technique (RDT) where prompt gamma radiation measured at the target position is correlated with (i) the specific mass/charge ratio of a residue (as inferred from the position at which the recoil crosses the FMA focal plane) and (ii) the subsequent characteristic alpha decay (measured with a Double-Sided Si Strip detector). A detailed account of these experiments can be found in Refs. [7] and [8].

Fig. 1 compares the $\gamma$ spectra obtained with the ${ }^{208} \mathrm{~Pb}\left({ }^{48} \mathrm{Ca}, 2 \mathrm{n}\right){ }^{254}$ No reaction at beam energies of 215 and $219 \mathrm{MeV}$. Transitions from higher-spin members of the groundstate band are clearly enhanced at the higher bombarding energy, and the decay of levels with spin as high as $20 \hbar$ has been observed. At both energies, the high efficiency of Gammasphere enabled coincidence relationships to be verified between the transitions despite the small production cross sections ( $\mathrm{a}$ few $\mu \mathrm{b}$ ). The identification of a rotational band immediately establishes that ${ }^{254} \mathrm{No}$ is a deformed nucleus. An inset in Fig. 1 presents the $\Im^{(1)}$ moment of inertia as a function of the rotational frequency. The spins of the emitting states and the energies of the lowest levels, which were not detected because they decay almost entirely by internal conversion, were deduced using fits to this smooth curve based on the Harris parameterization. Furthermore, using expressions relating the $2^{+}$level energy with the $B(E 2)$ values of rotors and those relating the $B(E 2)$, quadrupole moment, and deformation, a quadrupole deformation parameter of $\beta=0.27(2)$ was deduced for ${ }^{254}$ No (see Ref. [7] for details). This value is in agreement with a value of 0.25 given by different macroscopic model calculations [9], and with values of 0.27 and 0.26 from HFB [10] and relativistic mean field [11] calculations, respectively. The moment of inertia is also an important quantity for theory to describe as it is sensitive to the single-particle energies and pairing. HFB calculations of this quantity with both the 


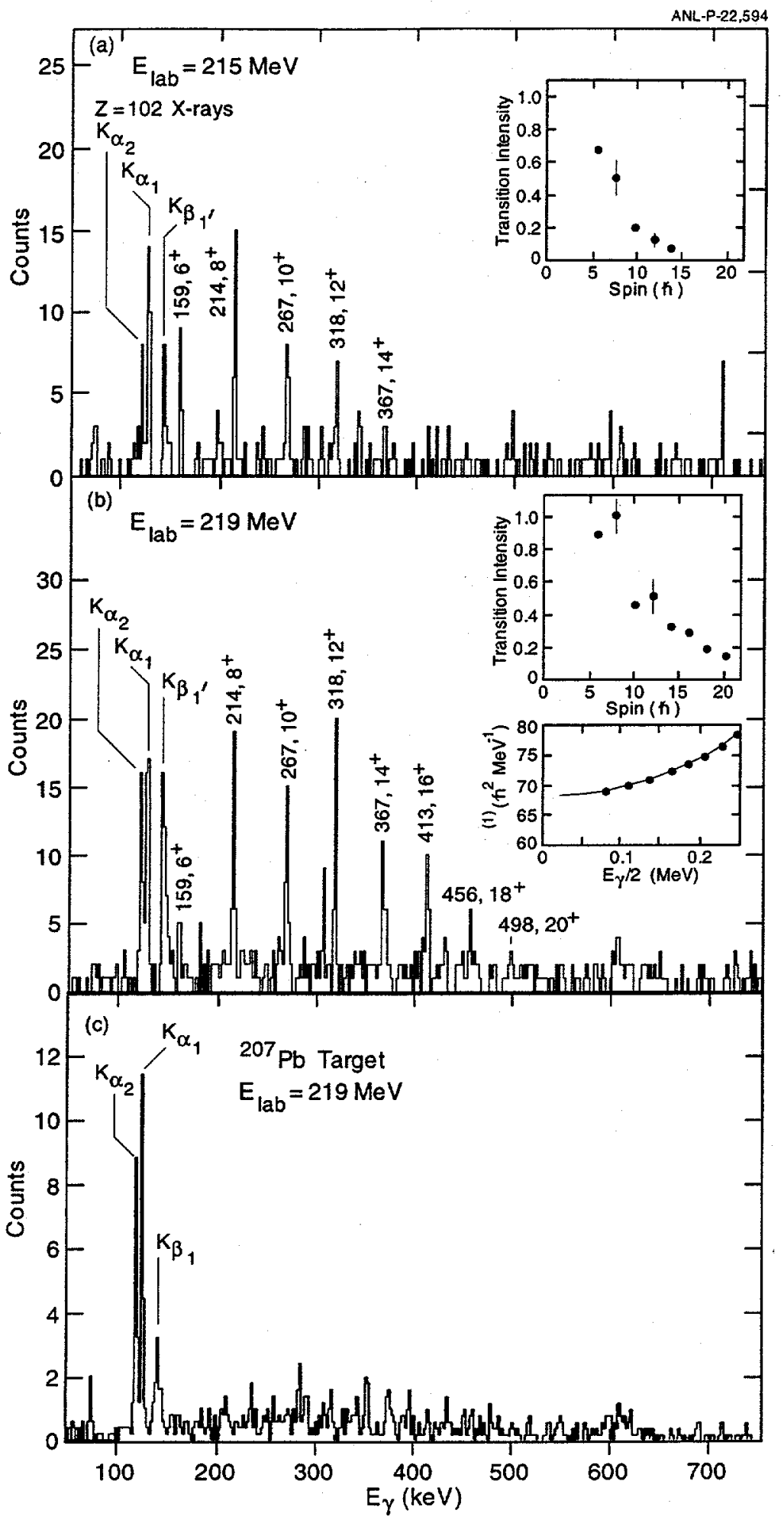

Figure 1. (a) and (b) ${ }^{254}$ No spectra obtained at two beam energies. The transitions are labeled by their energies and initials spins. The intensity profiles are given as insets. A second inset in (b) shows the evolution with frequency of the moment of inertia. The spectrum obtained for ${ }^{253}$ No is shown at the bottom. See Refs. $[7,8]$ for further details. 
SLy4 [12] and the Gogny [13] forces have recently become available. They reproduce the evolution of the moment of inertia with spin satisfactorily and assign the smooth increase with frequency to the gradual alignment of $i_{13 / 2}$ protons and $j_{15 / 2}$ neutrons. More recently, the nucleus ${ }^{253}$ No was studied with the ${ }^{207} \mathrm{~Pb}\left({ }^{48} \mathrm{Ca}, 2 \mathrm{n}\right)$ at $219 \mathrm{MeV}$ with the objective of providing more information on the behavior of neutron orbitals located in the vicinity of the Fermi surface. The results of this experiment are still preliminary. As can be seen in the bottom panel of Fig.1, the spectrum measured in coincidence with $A=253$ residues is quite different from those presented for ${ }^{254}$ No: the No $\mathrm{x}$-rays dominate the spectrum and an appreciable number of weaker $\gamma$ transitions are present at higher energies. These observations should not be viewed as a surprise. Electron-capture decay measurements into the isotone ${ }^{249} \mathrm{Cf}\left[14\right.$ ] and alpha decay studies into ${ }^{253}$ No itself [15] have shown that a number of neutron excitations occur within $\sim 500 \mathrm{keV}$ of the groundstate. Hence, several collective band structures can be expected to receive appreciable population in the reaction. In addition, the deexcitation of the states in these bands will be characterized by a competition between $\mathrm{M} 1(\mathrm{I} \rightarrow \mathrm{I}-1)$ and $\mathrm{E} 2(\mathrm{I} \rightarrow \mathrm{I}-2)$ transitions. The M1 transitions will be highly converted, as will the lowest energy E2 $\gamma$ rays. The competition between the two deexcitation modes depends on the intrinsic structure of the neutron orbitals and only excitations built on orbitals where the $B(\mathrm{M} 1)$ reduced probabilities are relatively small can be expected to result in a rotational cascade dominated by E2 transitions with sufficient intensity to be detected by Gammasphere. A search for such structures has resulted in a positive signal. The analysis is, however, still in an early stage and more work is needed before a firm assignment can be made.

\subsection{Entry Distribution, Fission Barrier, and Formation Mechanism}

Taking advantage of the fact that Gammasphere covers most of the $4 \pi$ solid angle around the target, the entry distribution in angular momentum and excitation energy for the formation of ${ }^{254}$ No was determined at both beam energies from the number of detector modules that fired and the total energy emitted by $\gamma$ radiation in each event. The technique and the results are discussed in detail in Ref. [8]. The entry distributions are compared in Fig. 2 for the two beam energies. The one-dimensional spin and excitation energy distributions are given as well.

At the lower beam energy, it is the maximum allowable energy after neutron emission that imposes a $16 \hbar$ limit on the angular momentum and not the fission barrier. At the higher energy, states up to $22 \hbar$ and an excitation energy $E^{*}=8.5 \mathrm{MeV}$ are populated. Clearly, ${ }^{254}$ No can survive against fission up to these limits. Gamma decay to the ground state originates from the entry distribution, implying successful competition against fission. As described in detail in Ref. [8], these data provide a lower bound on the fission barrier $B_{f}$ : even at high spin $B_{f} \geq 5 \mathrm{MeV}$, a value which in turn corresponds to a constraint on the shell energy of $E_{\text {shell }} \geq 4 \mathrm{MeV}$ for spin values $I=12-22 \hbar$. The measured entry distribution contradicts the hypothesis that the shell-correction energy decreases to $1 / e$ of its zero spin value at spin 15 [16], and is in agreement with a much smaller decrease with angular momentum obtained in the HFB calculations of Ref. $[12,13]$.

An unexpected feature of the entry distributions of Fig. 2 is the sharp tilt angle with respect to the yrast line. This is due, at least in part, to the small excitation energy of the low-spin entry states, which appears to be a distinct component of the distribution. 

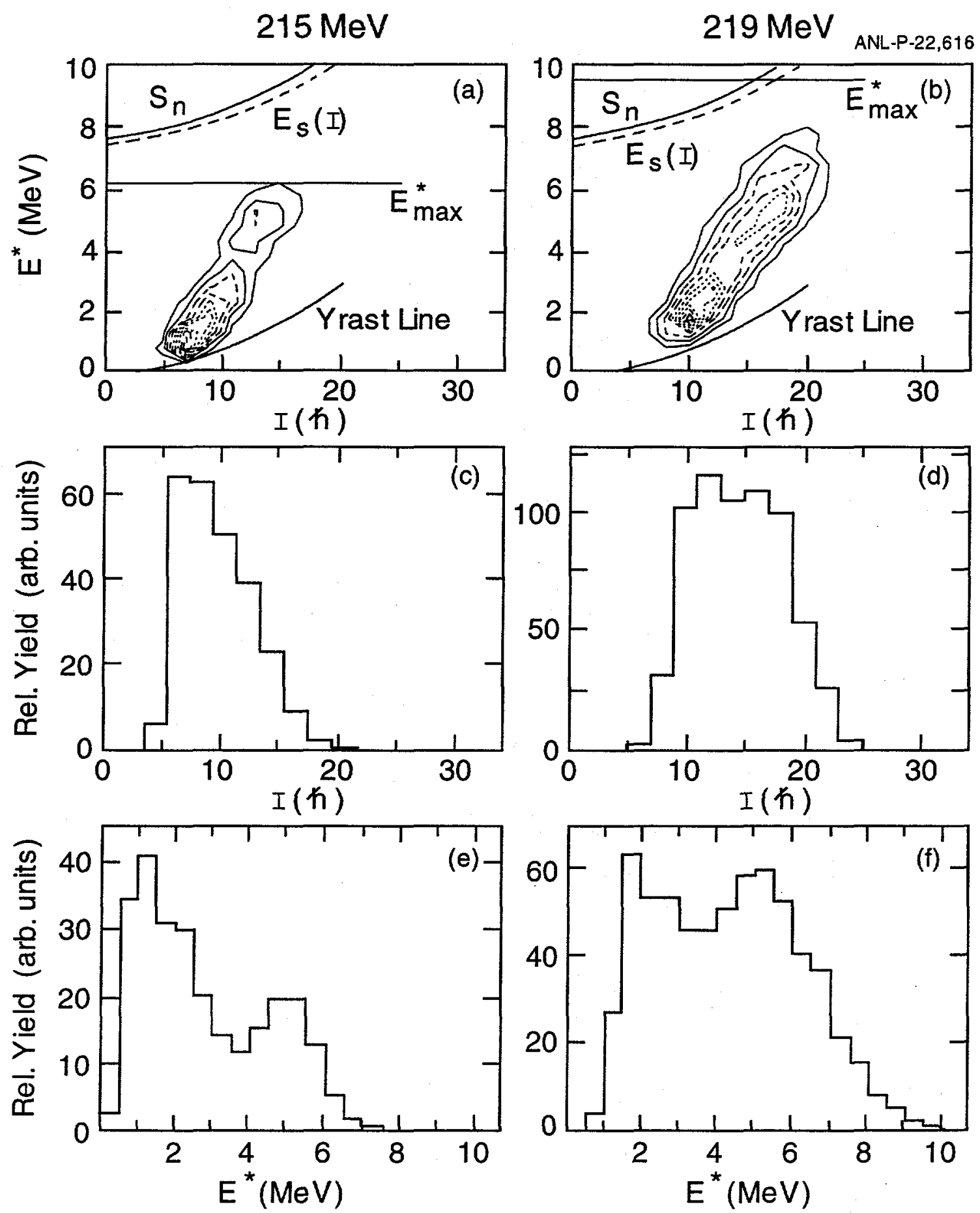

Figure 2. Contour plots of the entry distributions in spin and excitation energy and their projections at the two beam energies (left-215 MeV, right-219 MeV). The distributions in spin and excitation energy are also shown. Taken from Ref. [8]. 
The deviation of the entry distribution from the line representing $E^{*}{ }_{\max }$ gives the energy removed by the two neutrons, and the evaporation residues with low partial waves seem to be associated with unusually energetic neutrons. On the other hand, at higher spin ( $I \geq$ $14 \hbar$ ) the entry distributions above the yrast line are more usual. Hence, there is a hint of at least two mechanisms in the formation of these very heavy nuclei: a normal, statistical one responsible for high spin formation and another one with emission of higher-energy (perhaps preequilibrium) neutrons, which is important at lower spins. It is worth noting that the preliminary analysis of the ${ }^{253}$ No entry distribution confirms the conclusions given here for ${ }^{254}$ No.

\section{OCTUPOLE CORRELATIONS IN THE ACTINIDES}

In most nuclei of the rare earth and actinide regions, rotational bands of states with odd spins and negative parity appear at excitation energies of $\leq 1 \mathrm{MeV}$ which are interpreted as structures based on one-phonon octupole vibrations[17,18]. In nuclei where these correlations are much stronger, these negative parity states lie even lower in excitation energy, and stable octupole deformation may occur. In nuclei around ${ }^{146} \mathrm{Ba}$ and ${ }^{224} \mathrm{Th}$, bands with levels of alternating spin and parity, connected by strong electric-dipole transitions, represent the best experimental evidence for the rotation of octupole-deformed nuclei. It has been shown empirically that rotation can stabilize the octupole shape[17-19].

Recent progress in the study of actinide nuclei has come from work by Ward et al [20] who demonstrated the power of inelastic scattering at beam energies slightly above the Coulomb barrier on thick/backed targets as a means of studying collective excitations with high sensitivity: this is the so-called "unsafe Coulex" technique. At these beam energies the cross sections for feeding the highest spin states are enhanced. Under such conditions, most of the $\gamma$ rays are emitted after the excited nucleus has come to rest, and the vast majority of transitions in a collective cascade are measured with the intrinsic resolution of the Ge detectors. With Gammasphere at ATLAS, data on all $\mathrm{Pu}$ nuclei with mass $\mathrm{A}=238-244$, on ${ }^{237} \mathrm{~Np},{ }^{241} \mathrm{Am},{ }^{242} \mathrm{Cm}$ and ${ }^{246,248} \mathrm{Cm}$ were obtained from measurements performed with either ${ }^{207} \mathrm{~Pb},{ }^{208} \mathrm{~Pb}$ or ${ }^{209} \mathrm{Bi}$ beams at energies $\sim 15 \%$ above the Coulomb barrier. Details about the experimental techniques are presented in Refs. [21-23]. The level schemes of all target nuclei were considerably expanded. Those of nuclei reached via transfer reactions are less extensive, but provide important information nevertheless.

Figure 3 compares the aligned spins $i_{x}$ as a function of rotational frequency $\hbar \omega$ for the yrast sequences and for the lowest negative parity cascades in ${ }^{238-244} \mathrm{Pu}$. A number of interesting features clearly stand out: (i) all the $\mathrm{Pu}$ isotopes with mass $\mathrm{A} \geq 241$ exhibit a strong alignment in their respective yrast bands at $\hbar \omega \sim 0.25 \mathrm{MeV}$, (ii) this alignment is not present at all up to the highest frequencies observed in ${ }^{239} \mathrm{Pu}$ and ${ }^{240} \mathrm{Pu}$ and is delayed at least up to $\hbar \omega \geq 0.28 \mathrm{MeV}$ in ${ }^{238} \mathrm{Pu}$, (iii) the behavior of the alignment curve of ${ }^{240} \mathrm{Pu}$ is distinctly different from that of all the other even-even $\mathrm{Pu}$ isotopes, (iv) all negative parity excitations show the $\sim 2-3 \hbar$ alignment characteristic of the octupole phonon, and (v) an additional gain in alignment occurs only in the negative parity bands of the isotopes where a drastic upbend occurs along the yrast line, while, in contrast, (vi) the relative alignment between the two bands decreases in ${ }^{240} \mathrm{Pu}$.

The observation of sudden gains in $i_{x}$ of $9-10 \hbar$ in ${ }^{242} \mathrm{Pu}$ and ${ }^{244} \mathrm{Pu}$ complements the 


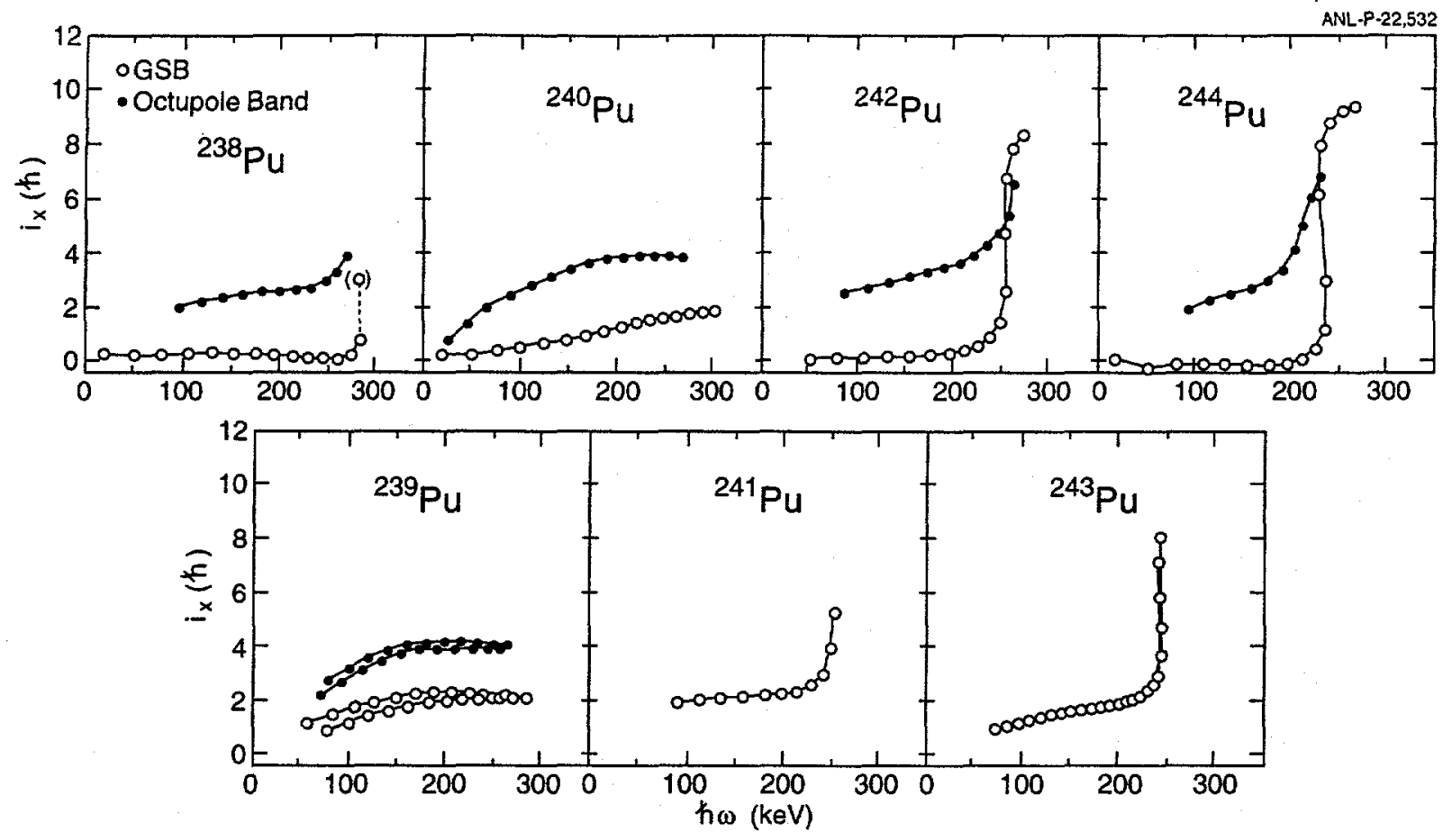

Figure 3. Aligned spins $i_{x}$ of the yrast and octupole rotational bands in the $\mathrm{Pu}$ isotopes. In all cases the same reference is subtracted, with the Harris parameters $J_{0}=65 \hbar^{2} \mathrm{MeV}^{-1}$ $J_{1}=369 \hbar^{4} \mathrm{MeV}^{-3}$. Taken from Ref. [23].

original findings of Ref.[24] by delineating completely, for the first time, a backbending in an actinide nucleus. This $i_{x}$ value is consistent with the alignment of a pair of $i_{13 / 2}$ protons, as predicted in Ref.[24-26]. The fact that the same alignment is also present in the odd- $\mathrm{A} \mathrm{Pu}$ isotopes and in the coupled bands based on the 5/2[523] proton orbital (of $h_{9 / 2}$ parentage), but not in the two bands built on the 5/2[642] configuration (of $i_{13 / 2}$ parentage) in ${ }^{241} \mathrm{Am}$ [27] agrees with this assignment (Pauli blocking). To the best of our knowledge, all the available calculations[24-26], indicate that the same strong proton alignment should occur around $\hbar \omega \sim 0.25 \mathrm{MeV}$ in the lighter Pu isotopes, yet this expected alignment gain is not seen in ${ }^{239,240} \mathrm{Pu}$ and is delayed in ${ }^{238} \mathrm{Pu}$. The effect is particularly striking in ${ }^{240} \mathrm{Pu}$ : only a small, smooth increase in alignment is observed over a range of 4-6 transitions beyond the point where the backbending occurs in the heavier Pu nuclei (Fig. 3). There is no sign of a band interaction neither in the yrast nor in the octupole band. Our recent data on ${ }^{242} \mathrm{Cm}$, the isotone of ${ }^{240} \mathrm{Pu}$, indicate the same absence of a band interaction along the yrast band of this nucleus[27]. In contrast, the band structures of another isotone, ${ }^{238} \mathrm{U}$, exhibit alignments similar to those seen in the heavier Pu nuclei[20].

In all the even-even Pu nuclei, the first excited band is of negative parity and is associated with an octupole vibration. This observation, together with the fact that octupole correlations are known to significantly alter alignment patterns seen in reflection symmetric nuclei[18,28], makes it worthwhile to search the data for additional indications of stronger octupole correlations near $A=240$. Fig. 4 presents the energy staggering, $S(I)$, between the odd-spin, negative parity and even-spin, positive parity bands. This stag- 


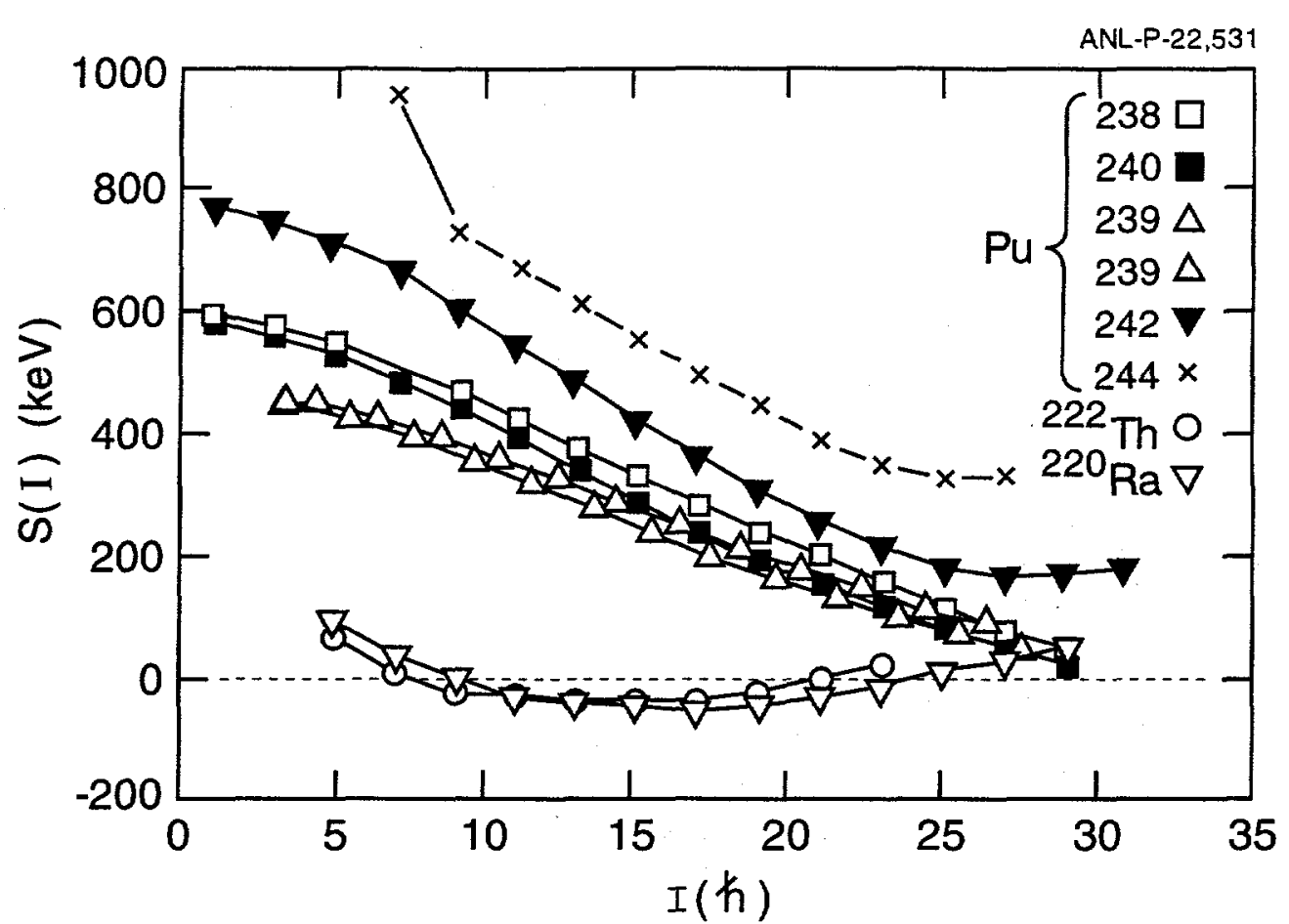

Figure 4. Comparison of the energy staggering $\mathrm{S}(\mathrm{I})=\mathrm{E}(\mathrm{I})-\left[(\mathrm{I}+1) \mathrm{E}_{(I-1)}+\mathrm{IE}_{(I+1)}\right] / 2 \mathrm{I}+1$ as a function of spin $\mathrm{I}$ in the $\mathrm{Pu}$ isotopes and in ${ }^{220} \mathrm{Ra}$ and ${ }^{222} \mathrm{Th}$, two of the best examples of octupole deformed nuclei.

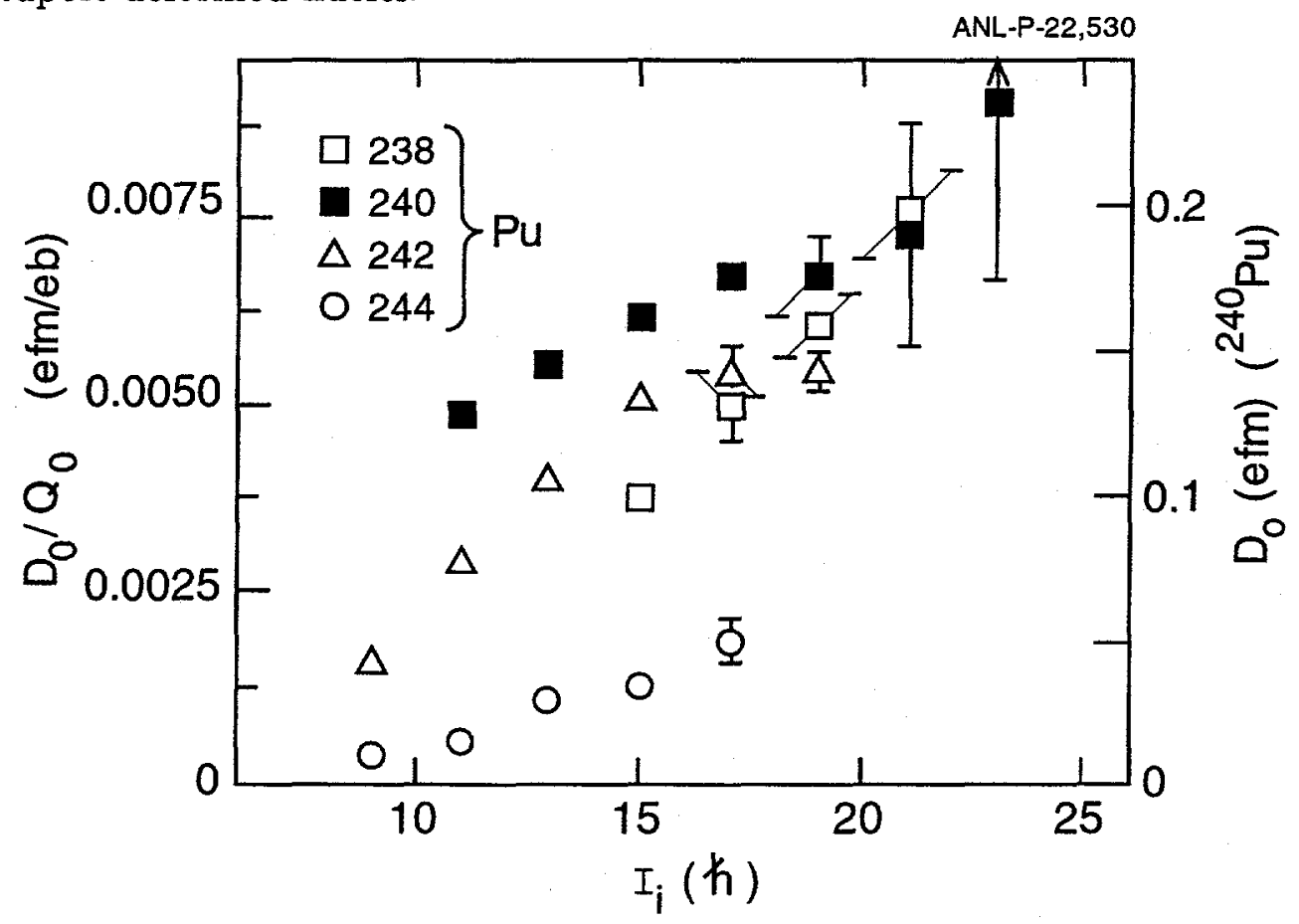

Figure 5. Ratio of transition dipole and quadrupole moments extracted from the E1 and E2 branchings $E 1: I_{i}^{-} \rightarrow\left(I_{i}-1\right)^{+} / E 2: I_{i}^{-} \rightarrow\left(I_{i}-2\right)^{-}$as a function of the spin $I_{i}$. The values of the transition dipole moment $D_{0}$ given on the right hand side are for ${ }^{240} \mathrm{Pu}$ only. They have been calculated assuming rotational E2-matrix elements with the $Q_{0}$ moment of the ground state band. 
gering is a measure of the extent to which the two bands of opposite parity can actually be regarded as a single, rotational octupole excitation. Remarkably, the $\mathrm{S}(\mathrm{I})$ values for ${ }^{238,240} \mathrm{Pu}$ decrease up to the highest spins, where they become small and comparable to those seen in ${ }^{222} \mathrm{Th}$ and ${ }^{220} \mathrm{Ra}$ [29], two of the best examples of nuclei with static octupole deformation. Moreover, the interleaving at high spin of states with opposite parity is also realized in the odd ${ }^{239} \mathrm{Pu}$ nucleus. Also, levels with the same spin but opposite parity are located close in energy: the $49 / 2^{+}$and the $49 / 2^{-}$levels are $17 \mathrm{keV}$ apart, the two $53 / 2$ states are separated by only $8 \mathrm{keV}$ and the $57 / 2$ levels are within $26 \mathrm{keV}$. Hence, these states appear to form so-called parity doublets, as expected for odd nuclei with static octupole deformation $[17,18]$. Thus, at least from the point of view of the level energies, the three lightest $\mathrm{Pu}$ isotopes behave like octupole deformed rotors at the highest spins. It is worth pointing out that the decrease in relative alignment between the two bands in ${ }^{240} \mathrm{Pu}$ noted above is consistent with this interpretation, although it suggests that the transition towards stable octupole deformation is not complete as the relative alignment is not yet zero.

Further indications about the importance of the octupole correlations come from the ratio of the transition dipole $\left(D_{0}\right)$ and quadrupole $\left(Q_{0}\right)$ moments shown in Fig. 5 for the even $\mathrm{Pu}$ isotopes. From the figure, it is clear that the strength of the E1 transitions grows with smaller mass number, as expected for the increase in the strength of octupole correlations. Furthermore, the out-of-band E1 decays become increasingly competitive with the in-band $\mathrm{E} 2$ decays as the spin in the negative parity band increases. The effect is most pronounced for ${ }^{240} \mathrm{Pu}$ and ${ }^{238} \mathrm{Pu}$. Under the assumption that the $\mathrm{B}(\mathrm{E} 2)$ values are constant within a rotational band, this result suggests an increase in E1 (i.e octupole) collectivity with angular momentum. Values at each spin for the induced intrinsic dipole moment $D_{0}$ have been extracted in ${ }^{240} \mathrm{Pu}$, assuming a constant quadrupole moment $Q_{0}=$ $25.9 \mathrm{eb}$ adopted from the measured $\mathrm{B}(\mathrm{E} 2)$ value of the $2^{+} \rightarrow 0^{+}$ground state transition [30]. It can be seen from Fig. 5 (right hand vertical axis) that the $D_{0}$ values at high spin become quite large; $D_{0} \sim 0.2 \mathrm{efm}$ for $\mathrm{I} \geq 21 \hbar$ in ${ }^{240} \mathrm{Pu}$. Such values are of the same order as the dipole moments observed in the light Th nuclei[31] $\left(D_{0} \sim 0.2-0.3 \mathrm{efm}\right)$, which are among the best examples of octupole deformed nuclei [18].

Finally, two other experimental observations point towards the importance of octupole correlations in the light Pu isotopes. As discussed recently by Sheline and Riley [32], the hindrance factors for alpha decay in the light $\mathrm{Pu}$ isotopes are smaller than those seen in all neighboring nuclei and are of the same order as the values measured in the octupole deformed Ra, Rn and Th nuclei. In ${ }^{240} \mathrm{Pu}$, strong $\mathrm{E} 1$ transitions linking members of the second-excited $0^{+}$band to the negative parity band have also been observed [33]: the measured $\mathrm{B}(\mathrm{E} 1) / \mathrm{B}(\mathrm{E} 2)$ ratios are of the same order than those seen in Fig. 5.

From the discussion above it is clear that the lightest $\mathrm{Pu}$ isotopes exhibit surprising effects associated with strong octupole correlations. Their impact translates in the absence $\left({ }^{239,240} \mathrm{Pu}\right)$ or delay in frequency $\left({ }^{238} \mathrm{Pu}\right)$ of the strong proton alignment seen in the heavier $\mathrm{Pu}$ nuclei. Furthermore, at the highest spins, the correlations are such that the yrast and the octupole bands merge into a single sequence of levels with alternating spin and parity, large intrinsic dipole moments can be inferred from the $\mathrm{B}(\mathrm{E} 1) / \mathrm{B}(\mathrm{E} 2)$ ratios, and so-called parity-doublets occur in ${ }^{239} \mathrm{Pu}$. The experimental evidence suggests that a transition from an octupole vibration to stable octupole deformation may have occurred. Such an evolu- 
tion with angular momentum has been predicted in Refs. [34,35]. Detailed microscopic calculations are needed to fully account for the enhanced importance of octupole correlations near ${ }^{240} \mathrm{Pu}$. In particular, the role of the octupole-driving orbitals needs to be fully explored. In this context, it is striking that in the immediate odd-even neighbors of ${ }^{240} \mathrm{Pu}$ the $\Delta l=3, \Delta \Omega=0$ particle-hole configurations $\pi\left\{3 / 2^{-}[521] 3 / 2^{+}[651]^{-1}\right\}$ and $\nu\left\{7 / 2^{+}[624] 7 / 2^{-}[743]^{-1}\right\}$ come close in energy to the Fermi surface (within $0.5 \mathrm{MeV}$ ). They are expected to play a significant role in the issues under discussion.

\section{CONCLUSIONS}

This presentation has focused on recent studies of actinide and transfermium nuclei performed with Gammasphere at ATLAS. In the Nobelium nuclei, the anticipated shape driving of the shell effects was confirmed. These heavy nuclei were found to be more stable against fission than had previously been thought. In addition, the measured entry distributions indicate that high partial waves contribute to the fusion cross section and that there may be more than one reaction mechanism. These results may well have implications for the synthesis of super-heavy elements. In the actinide nuclei, the strength of the octupole correlations near ${ }^{240} \mathrm{Pu}$ appears to be such that, at the highest spins, ${ }^{238-240} \mathrm{Pu}$ exhibit properties associated with stable octupole deformation, suggesting that a transition with spin from a vibration to stable deformation may have occurred.

\section{ACKNOWLEDGMENTS}

The author wishes to thank his many colleagues who have contributed to the results presented in this contribution. They are too numerous to be all listed here. Special recognition is due to his colleagues at ANL, K. Abu-Saleem, I. Ahmad, M.P. Carpenter, C. Davids, T. L. Khoo, F.G. Kondev, T. Lauritsen, C.J. Lister, D. Nisius, P. Reiter, D. Seweryniak, S. Siem, J. Uusitalo and I. Wiedenhöver, without whom none of this work would have been possible. This work is supported by the U.S. Department of Energy, Nuclear Physics Division, under Contract No. W-31-109-ENG-38.

\section{REFERENCES}

1. I. Y. Lee, Nucl. Phys. A520, (1990) 641c.

2. C. N. Davids et al., Nucl. Instrum. Meth. Phys. Res. B70, (1992) 358.

3. Yu. Ts. Oganessian et al., Nature (London) 400, (1999) 242; Phys. Rev. Lett. 83, (1999) 3154.

4. V. Ninov et al., Phys. Rev. Lett. 83, (1999) 1104.

5. S.G. Nilsson et al., Nucl. Phys. A115, (1968) 545.

6. A. Sobiczewski, Phys. Part. Nuclei 25, (1994) 119.

7. P. Reiter et al., Phys. Rev. Lett. 82, (1999) 509.

8. P. Reiter al., Phys. Rev. Lett. 84, (2000) 3542.

9. Z. Patyl and A. Sobiczewski, Nucl. Phys. A533, (1991) 132; S. Cwiok et al., Nucl. Phys. A573, (1994) 356; P. Moller et al., At. Data Nucl. Data Tables 59, (1995) 185.

10. S. Cwiok et al., Nucl. Phys. A611, (1996) 211; W. Nazarewicz and P.-H. Heenen, private communication. 
11. G. Lalazissis et al., Nucl. Phys. A608, (1996) 202.

12. T. Duguet et al., Nucl. Phys. in press.

13. J. L. Egido and L. M. Robledo, Phys. Rev. Lett. 85, (2000) 1198.

14. I. Ahmad et al., Phys. Rev. C14, (1976) 218.

15. F.P. He $\beta$ berger et al, Z. Phys. A359, (1997) 415.

16. P. Ambruster, Proc. R.A. Welch Foundation, 41st Conf. on Chemical Research, The Transactinide Elements, Houston, TX, p. 231.

17. I. Ahmad and P.A. Butler, Annu. Rev. Nucl. Part. Sc. 43, (1993) 71, and references therein.

18. P.A. Butler and W. Nazarewicz, Rev. Mod. Phys. 68, (1996) 349, and references therein.

19. J.F.C. Cocks et al., Phys. Rev. Lett. 78, (1997) 2920.

20. D. Ward et al, Nucl.Phys. A600, (1996) 88.

21. G. Hackman et al, Phys.Rev. C57, (1998) R1059.

22. R.V.F. Janssens et al, Proc. Int. Conf. on Perspectives in Nuclear Physics, November 13-16 1998, Paradise Island, Bahamas, eds. J.H. Hamilton, H. K. Carter, and R. B. Piercey, World Scientific (1999),p. 47.

23. I. Wiedenhöver et al, Phys. Rev. Lett. 83, (1999) 2143.

24. W. Spreng et al., Phys. Rev. Lett. 51, (1983) 1522.

25. J. Dudek, W. Nazarewicz and Z. Szymanski, Phys. Scripta T5, (1983) 171 and references therein.

26. J.L. Egido and P. Ring, Nucl. Phys. A423, (1984) 93.

27. K. Abu Saleem et al., to be published.

28. S. Frauendorf and V. V. Pashkevich, Phys. Lett. B141, (1984) 23.

29. J.F. Smith et al., Phys. Rev. Lett. 75, (1995) 1050.

30. C.E. Bemis Jr. et al., Phys.Rev. C8, (1973) 1466.

31. P. Schuler et al., Phys. Lett. B174, (1986) 241.

32. R.K. Sheline and M.A. Riley, Phys. Rev. C61, (2000) 057301

33. I. Wiedenhöver et al, to be published.

34. R.V. Jolos and P. von Brentano, Phys. Rev. C49, (1994) R2301.

35. R.V. Jolos and P. von Brentano, Nucl. Phys. A587, (1995) 377. 